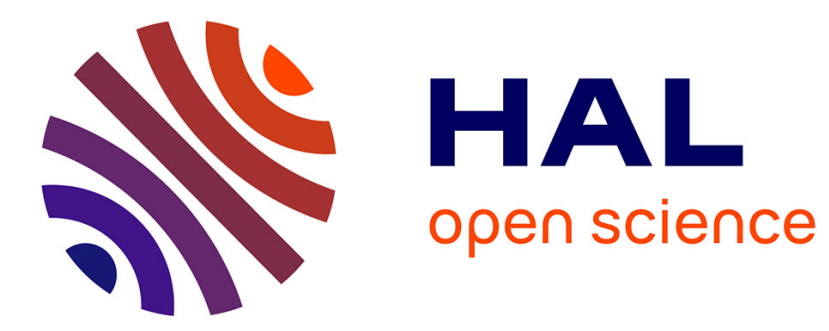

\title{
Touch without Touching: Overcoming Social Distancing in Semi-Intimate Relationships with SansTouch
}

Zhuoming Zhang, Jessalyn Alvina, Robin Héron, Stéphane Safin, Françoise

Detienne, Eric Lecolinet

\section{- To cite this version:}

Zhuoming Zhang, Jessalyn Alvina, Robin Héron, Stéphane Safin, Françoise Detienne, et al.. Touch without Touching: Overcoming Social Distancing in Semi-Intimate Relationships with SansTouch. CHI '21: CHI Conference on Human Factors in Computing Systems, May 2021, Yokohama (virtual), Japan. pp.1-13, 10.1145/3411764.3445612 . hal-03264979

\section{HAL Id: hal-03264979 \\ https://hal.science/hal-03264979}

Submitted on 18 Jun 2021

HAL is a multi-disciplinary open access archive for the deposit and dissemination of scientific research documents, whether they are published or not. The documents may come from teaching and research institutions in France or abroad, or from public or private research centers.
L'archive ouverte pluridisciplinaire HAL, est destinée au dépôt et à la diffusion de documents scientifiques de niveau recherche, publiés ou non, émanant des établissements d'enseignement et de recherche français ou étrangers, des laboratoires publics ou privés. 
archives-ouvertes

\section{Touch without Touching: Overcoming Social Distancing in Semi-Intimate Relationships with SansTouch}

Zhuoming Zhang, Jessalyn Alvina, Robin Héron, Stéphane Safin, Françoise Détienne, Eric Lecolinet

\section{To cite this version:}

Zhuoming Zhang, Jessalyn Alvina, Robin Héron, Stéphane Safin, Françoise Détienne, et al.. Touch without Touching: Overcoming Social Distancing in Semi-Intimate Relationships with SansTouch. CHI '21: CHI Conference on Human Factors in Computing Systems, May 2021, Yokohama Japan, Japan. pp.1-13, 10.1145/3411764.3445612 . hal-03264979

\section{HAL Id: hal-03264979 \\ https://hal.archives-ouvertes.fr/hal-03264979}

Submitted on 18 Jun 2021

HAL is a multi-disciplinary open access archive for the deposit and dissemination of scientific research documents, whether they are published or not. The documents may come from teaching and research institutions in France or abroad, or from public or private research centers.
L'archive ouverte pluridisciplinaire HAL, est destinée au dépôt et à la diffusion de documents scientifiques de niveau recherche, publiés ou non, émanant des établissements d'enseignement et de recherche français ou étrangers, des laboratoires publics ou privés. 


\section{Touch without Touching: Overcoming Social Distancing in Semi-Intimate Relationships with SansTouch}

\author{
Zhuoming Zhang \\ zhuoming.zhang@telecom-paris.fr \\ LCTI, Télécom Paris, Institut \\ Polytechnique de Paris \\ Paris, France
}

Stéphane Safin

stephane.safin@telecom-paris.fr

i3 UMR 9217-CNRS, Télécom Paris, Institut Polytechnique de Paris

Paris, France

\author{
Jessalyn Alvina \\ jessalyn.alvina@telecom-paris.fr \\ LCTI, Télécom Paris, Institut \\ Polytechnique de Paris \\ Paris, France
}

Françoise Détienne

francoise.detienne@telecom-paris.fr

i3 UMR 9217-CNRS, Télécom Paris,

Institut Polytechnique de Paris

Paris, France

\author{
Robin Héron \\ robin.heron@telecom-paris.fr \\ i3 UMR 9217-CNRS, Télécom Paris, \\ Institut Polytechnique de Paris \\ Paris, France
}

Eric Lecolinet

eric.lecolinet@telecom-paris.fr

LCTI, Télécom Paris, Institut

Polytechnique de Paris

Paris, France
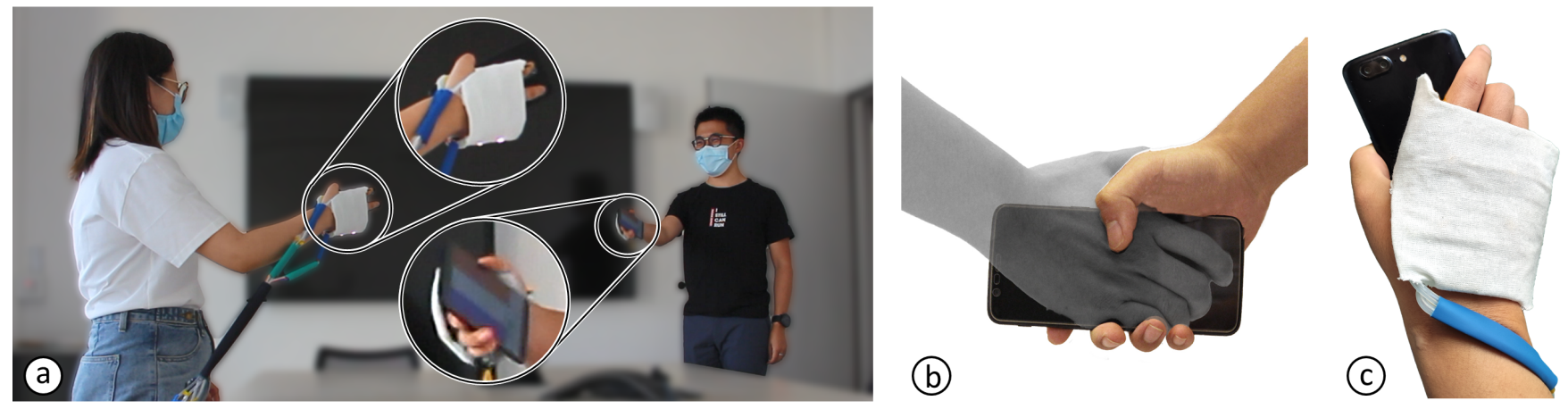

Figure 1: (a) Two users exchanging a mediated handshake with SansTouch at 2-meter distance. (b) To trigger the handshake stimuli, both users synchronously mimic the hand movement of a handshake as in real life. (c) Each user wears the multimodal hand device while holding a smartphone.

\begin{abstract}
Social distancing may force people to restrict social touch practices. Our survey $(\mathrm{N}=136)$ highlighted substantial social touch breakdowns during the COVID-19 pandemic for semi-intimate relationships (e.g., friends, colleagues), with handshakes being the most reduced, and frustrations at having to re-establish social touch habits. We then designed SansTouch, a multi-modal hand sleeve used together along with a smartphone to enable mediated hand-tohand interactions such as handshakes or holding hands. To invoke the mediated touch, users synchronously mimic the hand position as in real life while holding SansTouch. Users can feel the touch sensation in real time without touching. Participants from our observational study $(\mathrm{N}=12)$ quickly adopted the hand-to-hand
\end{abstract}

interactions of SansTouch for exchanging greetings face-to-face with colleagues and reported stronger preferences towards using SansTouch as opposed to mid-air gestures (e.g., waving). We discuss design implications, including the trade-offs of multi-modality for touch devices in face-to-face communication.

\section{CCS CONCEPTS}

- Human-centered computing $\rightarrow$ Haptic devices; Empirical studies in $\mathrm{HCI}$.

\section{KEYWORDS}

Mediated touch device, interpersonal touch communication, survey, interview

ACM Reference Format:

Zhuoming Zhang, Jessalyn Alvina, Robin Héron, Stéphane Safin, Françoise Détienne, and Eric Lecolinet. 2021. Touch without Touching: Overcoming Social Distancing in Semi-Intimate Relationships with SansTouch. In CHI Conference on Human Factors in Computing Systems (CHI '21), May 8-13, 2021, Yokohama, Japan. ACM, New York, NY, USA, 13 pages. https://doi.org/ $10.1145 / 3411764.3445612$ 


\section{INTRODUCTION}

Social touch is an essential non-verbal communication channel that is widely used for different purposes, from expressing less intimate feelings such as greetings (e.g., handshakes) to more intimate ones such as kissing or hugging [47]. However, social touch communications can be highly restricted under certain circumstances, for instance due to social distancing during a pandemic like COVID-19 [8] or for people with medical conditions [21, 39]. In these circumstances, even when people are physically in the same place (i.e., face to face), social touch breakdowns may still occur: they may not be able to directly touch each other like they used to. Moreover, the social touch breakdowns may not only have direct consequences on intimate relationships such as families or close friends, but also on semi-intimate relationships such as casual friends or colleagues. Research in other domains highlighted that long-term touch deprivations may severely impact health and well beings $[13,20]$. As such, it is important to overcome these social touch breakdowns, especially under circumstances in which people might have to practice social distancing for a long time [8].

Although research in $\mathrm{HCI}$ have proposed different technologies to mediate interpersonal touch communication, these touch devices were mainly designed for intimate relationships such as couples (e.g., touching cheeks [36], kissing [40]) and close friends or families (e.g., hugging [1, 3, 21], tickling [15]). Research in robotics have proposed robot arms to enable remote handshaking for semi-intimate relationships like colleagues [31]. However, robot-mediated touch devices often suffer from uncanny valley issues ${ }^{1}$ [31]. Furthermore, these touch devices were mainly designed for and evaluated in the context of remote communications [17, 28, 31, 35, 48]. As such, their effectiveness and applicability when used during face-to-face communications remain unclear, especially since past research suggested that establishing face-to-face communications introduces different challenges compared to remote communications [6].

Our work aims to complement and expand the design space of computer-mediated touch, to better support interpersonal touch communications that are: 1) not only for users with intimate but also semi-intimate relationships, and 2) not only used in remote but also face-to-face communications. Additionally, we aim to build a novel touch device that can generate realistic touch sensations.

To better inquire into the potential use case scenarios of touch devices in face-to-face communications, we first conducted a survey with 136 participants (followed by in-depth interviews with 6 participants) to investigate social touch breakdowns due to social distancing during the COVID-19 pandemic. In particular, we were interested in the forms of social touch that were highly restricted with social distancing, the relationships that suffered from social touch breakdowns, and the challenges of re-establishing the alternative ways of touching others while social distancing. The survey revealed reduced social touch practices, in particular for greetings (e.g., handshakes) between people with semi-intimate relationships. Based on these insights, we built SansTouch, a multimodal hand sleeve used along with a smartphone to enable handto-hand touch interactions without directly touching. SansTouch

\footnotetext{
${ }^{1}$ Uncanny valley is when the emotional response to a device would abruptly shift from empathy to revulsion as it approached, but failed to attain, a lifelike appearance [30].
}

combined three modalities that were recommended in the literature to enhance touch sensations: skin-like inflation [31, 45], heat conditioned to body temperature $[3,17,31]$, and visual feedback $[15,50,53]$. We then conducted a structured observational study with 12 participants to 1) evaluate user perceptions and the use of SansTouch's handshakes in a face-to-face communication for semiintimate relationships (i.e., colleagues); and 2) elicit user feedback regarding the generalizability aspects of SansTouch, including the use of SansTouch's hand-to-hand interactions for other types of relationships and remote communications.

Our paper makes the following contributions: First, we contribute empirical insights based on real stories from participants on social touch habit breakdowns, including handshakes were the most reduced form of social touch, the frequencies of touch were highly reduced in semi-intimate relationships like casual friends and colleagues, and participants were frustrated having to learn new forms of social touch. Second, we propose SansTouch, a novel wearable hand sleeve used along with a smartphone, as well as the design of hand-to-hand interactions with SansTouch. The participants in our structured observation study reported high similarities of handshaking with SansTouch to a real handshake, and they could easily adopt the mediated touch interactions in a face-to-face conversation with others with minimum learning efforts. Finally, we discuss the design factors that can better support mediated touch interactions in both face-to-face and remote communications as future directions, including the trade-offs of combining different modalities, the level of synchronicity, and the social acceptability of the communication medium in different contexts of use.

\section{RELATED WORK}

As one of the major non-verbal channels in face-to-face communication, touch is widely used for conveying different information [47], from social etiquette like greetings (e.g., handshakes, kissing cheeks) to functional touch such as notification, punishment, and massaging [13]. Touch also influences human's physiology and psychology states as well as the social behavior [47]. For example, touch in intimate relationships (e.g., holding hands or hugs) could help lower pain, stress, and blood pressure [12, 19, 29], while touch in semi-intimate relationships could help reducing heart rate and stress level $[23,49]$. Touch deprivation might bring negative impacts on psychological state and well beings [13, 20,27]. Past research have also shown a linear correlation between affection deprivation (the lack of affective touch) and depression, stress, and emotional awareness for adults [14]. Given the importance of maintaining interpersonal touch, HCI researchers have proposed different mediated touch communication tools. In this section, we focus on discussing past research that specifically explored the role of touch devices in strengthening social connections and the design factors that are important to enhance touch sensations.

\subsection{Computer-mediated touch}

2.1.1 Robot-generated touch. In the field of human-robot interaction, technologies are often designed to generate touch with similar purposes of human touch. The touch stimuli are often pre-recorded and replayed to users. The touch experience is affected by the visual appearance, the perceived intent, and the physical qualities, for 
example users prefer touching soft, warm devices [3]. Calmer [21] was specifically designed to simulate maternal skin-to-skin holding for premature infants in incubators. Nakanishi et al. [31] coupled a robot hand and a video streaming to enable remote handshakes between two remote users. The robot hand was designed with a warm, skin-like material to imitate the sensation of touching a human hand. The handshake movement was robot-generated, although the replay was triggered by a user as soon as another user touched the robot hand. They highlighted the importance of synchronizing visual feedback and touch sensation. Although robot-generated touch was shown to strengthen remote social connections, it is often perceived as a "back-up" by users [21]. Furthermore, human-robot interaction often suffers from uncanny valley issues [31].

2.1.2 Interpersonal mediated touch. Past research have also explored two-way remote touch communications. DiSalvo et al. [11] built a doll-like hugging device that works in pairs to enable remote hugs. The device provides visual and audio feedback when its paired device is being hugged; and vibrates when its counterpart is being stroked. As such, the user understands that the stimuli are initiated by the other user simultaneously. Similarly, the iXu teddy bear device [26] moves its arms when its paired device is being stroked, mimicking the strokes of the other user. As such, the touch experience is bi-directional and shared between both users: both experience touching and being touched at the same time. To increase the perception of shared experience, KUSUGURI [15] not only delivers a tickling stimuli on the user's palm, but also displays the other user's finger as if it directly tickles the user's own palm.

These mediated touch interactions were mainly designed for users with intimate relationships like couples or close friends, and mostly evaluated in the context of remote communications [17, 28, 31, 35, 48]. However, special circumstances like social distancing may restrict interpersonal touch communications even when users are co-located, for example when interacting with fragile patients in hospitals [21,39] or during a pandemic [41] like the COVID-19 outbreak that became a global pandemic in early 2020 [2]. Existing research related to co-present mediated touch mainly focused on collaborative physical games [5] or augmenting direct touch experience in face-to-face communication $[1,51]$. In this case, the users could still touch each other. To the best of our knowledge, few considered the situation in which the users are face to face, can communicate through visual and auditory channels, but cannot touch each other. Our work attempts to fill in this gap, by exploring the possibilities of enhancing the design of touch devices to better support face-to-face communications.

\subsection{Designing mediated touch devices}

2.2.1 Communication medium. When designing a touch communication tool, it is critical to choose an appropriate communication medium. One option is using doll-like touch devices, as done in $[11,26]$. O'Brien et al. [32] compared several doll-like touch devices to represent the other person's hand in remote touch communications. However, their participants showed unwillingness to use doll-like touch devices while they were in public, sometimes even hid them in their bag or left them at home. This suggests that doll-like devices might have a low social acceptance level.
Another alternative is using wearable devices, for example tactile arm sleeves [53, 54], gloves [42], or jackets [10]). These fabricbased wearable devices were compact but expressive, enabling users to exchange affective touch interactions while preserving the possibility of natural interactions with their environment [34, 38]. Another advantage of using wearable devices is the touch stimuli can be delivered directly onto users' skin as soon as the stimuli is triggered, enabling highly synchronized communication that is critical for face-to-face communications [6].

2.2.2 Generating touch stimuli. Most touch devices used vibration motors to generate the touch stimuli. For example, Multi-Moji [50] used the built-in vibration motors in smartphones to enhance emojis with tactile feedback. TaSSt [24] and VisualTouch [53] used vibration motor arrays embedded on a tactile sleeve to generate tactile patterns on forearms. Similarly, Flex-N-Feel [42] embedded several vibration motors on a pair of gloves to support remote touch communication for couples. While vibration motors are easily available and relatively cheap, the touch sensation they can generate is quite limited and different from a real human touch [15].

Inflatable actuators offer more possibilities of generating realistic skin-like touch sensation, as it features a smaller difference in the mechanical impedance between the device and human [54]. For example, POKE [35, 37] used two inflatable bubbles attached on each side of a smartphone. Users can poke the inflatable bubbles as if poking the cheek of another person. Their longitudinal studies highlighted the different inflation patterns that the each couple developed over time [35]. Inflatable actuators have also been used to generate touch stimuli on arms [54], wrists [52], and torso [10]. Complementary but distinct from these devices, we proposed a novel touch device that embedded inflatable actuators on a wearable hand sleeve to enable hand-to-hand touch interactions.

Combining touch with other modalities could also expand the available range of emotional states and increase the touch perception [43, 50, 53]. For example, Wilson et al. [50] showed that combining visual and thermal modalities in touch interactions allowed users to convey a wider range of affective expressions. Tewell et al. [43] suggested that by adding a thermal array display (TAD) to text messaging systems, the emotional perception of the message could be enhanced. Adding an LED array that is activated along with a vibration motor array was shown to increase the touch perception [53]. Nevertheless, the combination of these modalities have not been evaluated in the context of face-to-face communications, thus its effectiveness for face-to-face communications remains unclear.

\section{STUDY 1: SURVEY ON SOCIAL TOUCH WITH SOCIAL DISTANCING}

Due to social distancing measures, people around the world have been forced to restrict and/or change their social touch habits even when they are co-located with the others, especially after COVID-19 pandemic lockdowns were imposed in their countries [4]. To better understand potential challenges and use case scenarios of mediated touch devices in face-to-face communication, we first wanted to investigate how the social distancing measures affected the different forms of social touch and the different types of social relationships. To this end, we conducted a survey on social touch habits before and after the lockdown during the COVID-19 pandemic to identify 
the social touch breakdowns for different social relationships and the alternatives replacing their touch habits.

\subsection{Recruitment and participants}

We recruited 136 participants (46\% women, 52\% men, $2 \%$ preferred not to say) who were at least 18 years old in different countries. More than half of them are adults below 30 years old (56\%). The rest are between $30-40$ years old (31\%), $40-49$ years old (4\%), and 50-59 years old (8\%). The effect of cultural backgrounds on social touch breakdowns was not our focus, so we did not specifically control the country of residence nor the cultural background as an independent variable in the participant pool. That said, to diversify our participant pool, we tried to include participants from different cultural backgrounds. Our participants were currently living in different continents: Europe (59\% of participants), Asia (36\%), America (4\%), and Australia (1\%). Although the majority of them lived in Europe, the participant pool included diverse cultural backgrounds: 58\% Asian, 37\% Europeans, 4\% Americans (i.e., north and south Americans), and 1\% Africans. Around one third of them (33\%) currently lived outside of their country of origins. When filling in the survey, the lockdown had ended for more than a month where the majority of the participants $(70 \%)$ lived.

\subsection{Method}

The participants completed the online survey in 15 minutes on average. The survey consisted of four parts. Part 1 collected demographic data including countries of origin and residency. Part 2 asked participants about their social touch habits before the lockdown started, while Part 3 about their new social touch habits after the lockdown ended. In these two parts, we asked about 1) the frequency of social touch (Likert-scale $1=$ "Never" to $5=$ "Always"); and 2) the forms of social touch (e.g., handshake, holding hands, hugs, kissing cheeks, etc.) for different relationship categories (Figure 2). We chose the relationship categories considering different intimacy levels and our pilot study with three participants. Specific to Part 3 , we asked two additional questions: 1) the forms of social touch that the participants wished to do but did not after the lockdown; and 2) frustration levels for their current social touch habits (Likert-scale $-2=$ "Extremely frustrated" to 2="Extremely happy"). In Part 4, we asked the participants to share social touch breakdown stories: a past event in which, during a face-to-face communication, they wanted to touch someone but could not due to social distancing (if any). For each story, the survey asked: 1) with whom; 2) the context (e.g., why they did not touch); 3) the form of social touch they wanted to do; 4) the purpose of social touch; and 5) what they did instead.

We also conducted follow-up interviews: we first went through the social touch breakdown stories from the survey, then contacted 8 participants with the richest stories, i.e., included concrete details related to social context and/or how they dealt with it (6 participants responded). This helped us to understand the nuances in the issues and the rationale behind their social touch decisions. These interviews lasted 20-45 minutes.

\subsection{Data Analysis}

We started the data analysis by performing statistics on the quantitative data (i.e., Likert-scale data) related to the evolution of the frequency and the forms of social touch before and after the social distancing. Although sharing social touch breakdown stories was optional, our survey collected a total of 84 stories reported by 72 participants (53\%). 82 stories were analyzed (we discarded 2 irrelevant stories e.g., due to long distance relationships). We analyzed the data inductively and deductively with several iterations using Braun and Clark's thematic analysis approach [7]. Altogether, the recurring themes highlighted different levels of social touch breakdowns for different social relationships, and the specific challenges that the participants faced as they re-established their new social touch habits.

\subsection{Key results}

3.4.1 Social touch breakdowns affected social relationships differently. Not surprisingly, some participants avoided seeing people in their circles even after the lockdown ended. Of those who met others after the lockdown ended, the participants reduced the frequency of touch with their colleagues the most (68\%), followed by close friends (64\%) and casual friends (61\%) (Figure 2). In particular, they were the most frustrated that they could not share social touches with close friends. The social touch breakdown stories were also dominated by wanting to touch casual friends (27\%) and close friends (17\%) but could not.

Around one third of the participants reported frustrations related to their new social touch habits with extended families (35\%) and nuclear families (31\%). Of particular interest, the extended family category had the highest number of participants reporting extremely frustrated (14\% of participants). This might be related to health concerns, as extended families most likely included the elderly who were more vulnerable to viral infections [8], as expressed by P93, "I have extended family members with health issues, and therefore avoid giving them hugs as much as possible when greeting them, even avoid touching them all together. It's depressing."

On the other hand, the social touch frequencies with partners, neighbours, and strangers in general remained the same. Only $18 \%$ of participants reported reduced frequencies for partners (the lowest), while only around $34 \%$ of participants for both neighbors and strangers. No social touch breakdown stories specifically mentioned wanting to touch their partners but could not. Hence, we might conclude that the social distancing significantly affected social touch among semi-intimate relationships such as casual friends and colleagues, but not intimate relationships (e.g., partners) nor strangers.

3.4.2 Handshakes, kissing cheeks, and hugs were highly reduced. Before the lockdown started, we found that the most frequent forms of social touch that the participants had had were handshakes, kissing cheeks, and hugs. Notably, both handshakes and kissing cheeks are commonly used as greetings. A high number of participants reported handshaking with all social relationships. Hugs were more likely shared among intimate relationships, as reported by the participants hugging partners, families, and close friends.

That said, we observed a significant decrease of the number of participants handshaking after the lockdown ended, especially with neighbours (from $57 \%$ to only $13 \%$ of participants), casual friends ( $51 \%$ to $19 \%$ ), close friends ( $43 \%$ to $24 \%$ ), extended families $(35 \%$ to $20 \%$ ), and colleagues (26\% to $8 \%$ ). Despite these decreases, they 


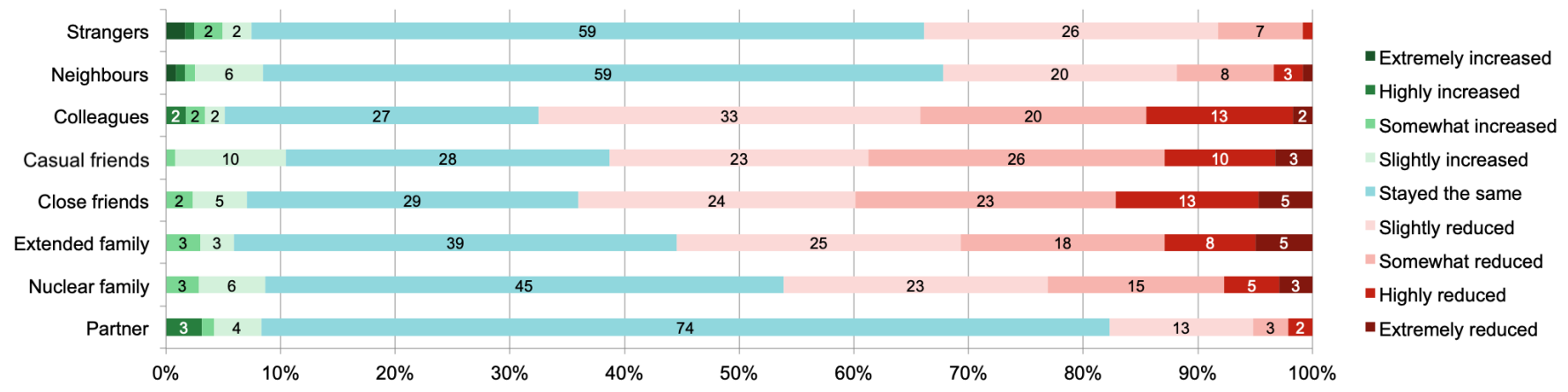

Figure 2: The percentage of participants who increased (green bars) or reduced (red bars) the frequency of social touch when they met after the lockdown ended. 'Extremely reduced' was when the participants reported 'always' touching before the lockdown but 'never' touching after the lockdown ended. The highest reduced frequency was with colleagues, while the frequency mostly remained the same for partners.

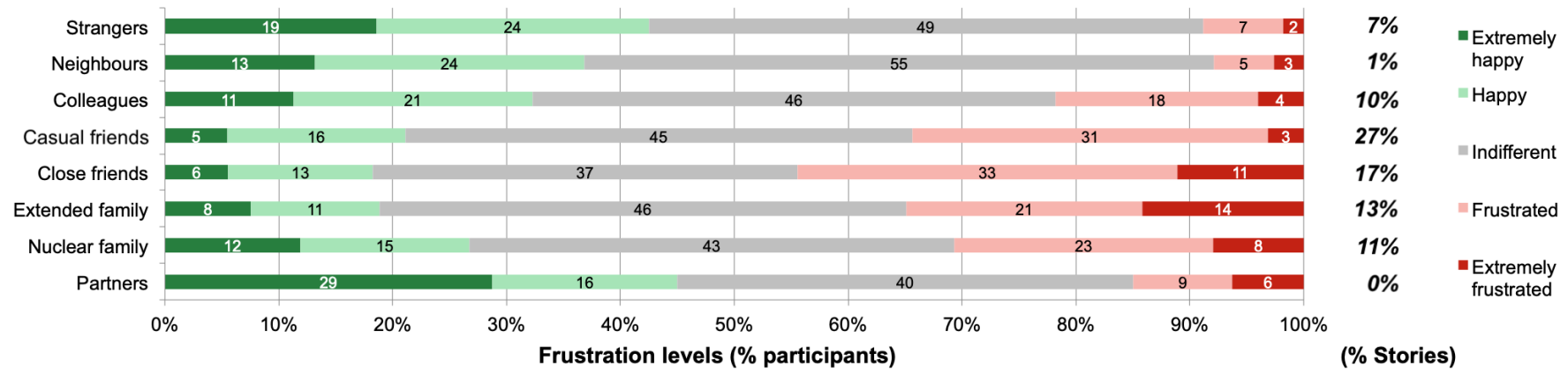

Figure 3: The perceived frustration levels of the new social touch habits and the percentage of social touch breakdown stories per relationship category reported by participants. Only $86 \%$ of the stories specifically mentioned with whom the social touch breakdown happened. High frustrations were reported for close and casual friends.

actually wished they could handshake other people, notably neighbours (43\%), friends (40\%), close friends (36\%), and colleagues $(31 \%)$. We also observed a similar trend of wanting to do it but could not for kissing cheeks and hugs. These results were in line with the social touch breakdown stories reported by the participants, with the majority of them expressing greetings (39\% of the stories), affections (24\%), and missing the other person (24\%). The forms of social touch that they wanted to have but could not included hugs $(37 \%$ of the stories), kissing cheeks (25\%), handshakes (18\%), touching hands $(6 \%)$, and touching face (1\%) (the rest was unspecified).

3.4.3 Social touch breakdowns forced the participants to re-establish touch communication habits. In more than half of the social touch breakdown stories, the participants ended up not touching the other person at all. In $37 \%$ of the stories, the participants substituted their old habits with other forms of social touch, such as midair gestures (e.g., waving, namaste, kiss bye). 19\% of the stories reported "inventing" new forms like bumping fists, knees, feet, elbows, and arms. Given these different new social touch habits, some participants reported confusions and frustrations as they were not sure what forms of social touch were acceptable anymore and having to learn the new forms of social touch. $11 \%$ of the social touch breakdown stories specifically described this confusion. P51 explained that he had to observe the body gestures of the person he was talking to, in order to guess which form of social touch the other person would use for greetings. P87 also felt that this now-necessary observation was frustrating, especially if the other person was not familiar with the new form: "It is annoying now I have to read the signs of what each person wants to do. One time, I was like, heyyy, and lifted my elbow [to do an elbow bump] and then yeaahhh, she didn't understand [what I was doing], so I had to turn it into something else. It was super awkward."

To avoid this confusion, some participants had to explicitly negotiate on the forms of social touch they were going to share, as described in $24 \%$ of the stories. However, this situation was often perceived as uncomfortable by the participants. For example, P42 had to reject a handshake but was worried that the other person would misunderstand: "I said, 'I'm sorry but no handshakes, let's do this instead', like namaste, or fist bumps. I was trying not to hurt their feelings. It was very hard to say something like that to my colleagues or close friends."

\subsection{Discussion}

Reflecting back on our goal to design a novel touch device that supports face-to-face communications, we highlighted three of the survey findings that served as challenges and opportunities for 


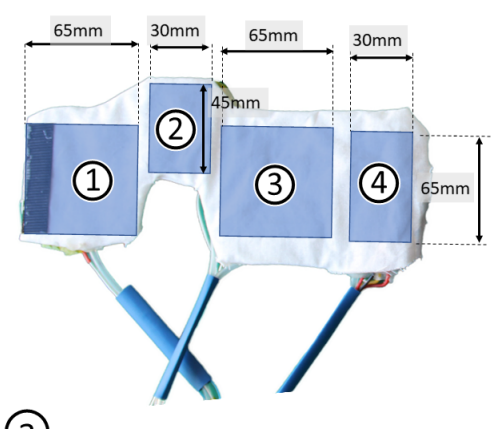

(a)

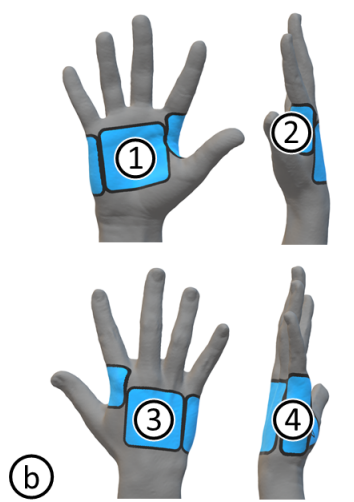

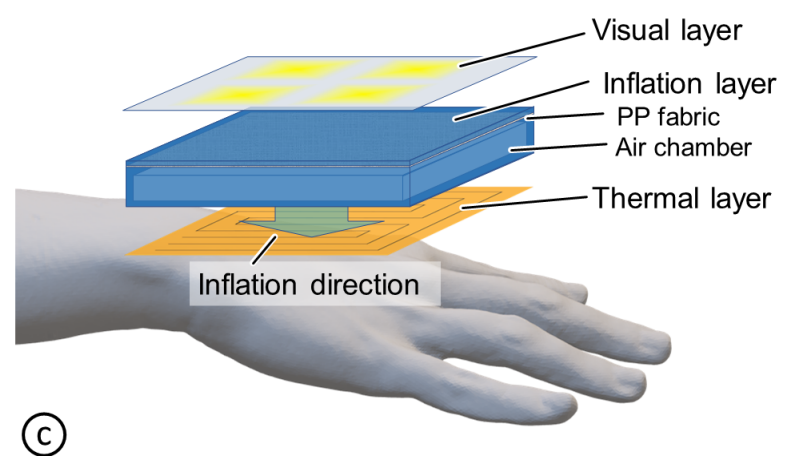

Figure 4: (a) SansTouch's output channel is a wearable hand sleeve. (b) It consists of four patches located on the palm, the back of the hand, and the two sides of the hand. (c) Each patch consists of three layers: visual, inflation, and thermal.

design: First, social touch practices were the most reduced between people with semi-intimate relationships like casual friends and colleagues. This finding emphasized the need to design and evaluate mediated touch communication tools for semi-intimate relationships, which are currently under-explored in the literature. Second, handshakes, kissing cheeks, and hugs were highly reduced after the lockdown - the forms of social touch commonly used for greetings. This ritualistic touch is an important part of our daily touch behaviour [25], and failing to greet another person appropriately may have consequences for the embodied relationships [27]. This result highlighted the opportunities to design mediated touch devices for handshakes, complementing the past research on mediated touch devices for hugs $[1,11,26]$ and for cheeks $[35,36]$. Finally, the participants reported struggles and frustrations, not only because their social touch habits no longer worked (i.e., social touch breakdown), but also they had to establish new social touch habits with extra learning efforts. Establishing communication between two people requires a common understanding (i.e., a common ground) on the purpose and the medium of the communication [6]. Hence, when the participants tried to replace the touch with mid-air gestures (e.g., waving hands) or "inventing" new form of greetings (e.g., fist or elbow bump), they faced some challenges updating and negotiating their common ground with the other person, which they perceived as frustrating. These results motivated us to explore the possibilities of using mediated touch device to re-enable some common forms of greetings, such as handshakes, that can be used in face-to-face communications.

\section{SANSTOUCH: ENABLING REMOTE HAND-TO-HAND INTERACTIONS}

\subsection{Design rationale}

Our goals were to build a novel touch device that 1) enables users to exchange bi-directional touch for greetings without physical contacts in face-to-face communications; 2) minimizes the efforts to learn new touch interactions; and 3) can generate realistic touch sensation (i.e., similar to the real touch). The design process included two steps: designing the touch communication medium and designing the user-to-user interactions. We considered that interpersonal touch communications should be bi-directional: users feel the sensation of touching and being touched at the same time. Considering these aspects, we proposed SansTouch, a mediated touch communication tool that combines a wearable hand sleeve and a smartphone (Figure 1). The wearable device of SansTouch is a hand sleeve wrapping on the hand of user (Figure 4, further described in Section 4.2). Considering the social acceptability aspect [32], we opted for smartphones to represent the other person's hand as they have a high social acceptance, high accessibility, built-in sensors, as well as connectivity features. Since they are handheld devices, usually users can comfortably grip their smartphones as well.

With SansTouch, we can emulate handshakes and other handto-hand interactions such as holding hands, high-fives, or patting hands (Figure 6). The interactions happen mid air, with both the sender and the receiver wearing the wearable hand device while holding the smartphone. The touch stimulus is triggered as soon as both users synchronously mimic the hand position as in real life. We specifically let users mimic the real hand interaction to minimize the efforts to learn the mediated interactions and to establish the communication ground with SansTouch.

\subsection{Generating touch stimuli with SansTouch}

The output channel of SansTouch is a wearable sleeve wrapped on the user's hand that can generate a warm touch along with visual feedback. SansTouch has four different patches (Figure 4A and B): one patch on the palm, one patch on the back of the hand (Figure 4a (1),(3)), and two patches on the sides of the hand (Figure 4a (2),(4)). Each patch consists of three layers: thermal, inflation, and visual (see Figure 4c).

4.2.1 Skin-like grip force. Our goal is to reproduce skin-like touch sensations that could be perceived as more realistic than those generated with vibration motors. As in [31, 36, 45], we opted for skin-safe and highly stretchable silicon rubbers (ref. Ecoflex 00-20) to make inflatable airbags that generate a grip force on the user's hand. The walls of the silicon airbags are $1.5 \mathrm{~mm}$ thick, while the surfaces are $3 \mathrm{~mm}$ thick, with a layer of polypropylene (PP) fabric inserted to limit its stretchability. With this setting, the airbag mainly inflates towards the skin, increasing the generated touch pressures. The inflation and deflation of each airbag is controlled by 2 two-way DC solenoid valve (ref. ZHV0519) through a 16 channel PWM servo 

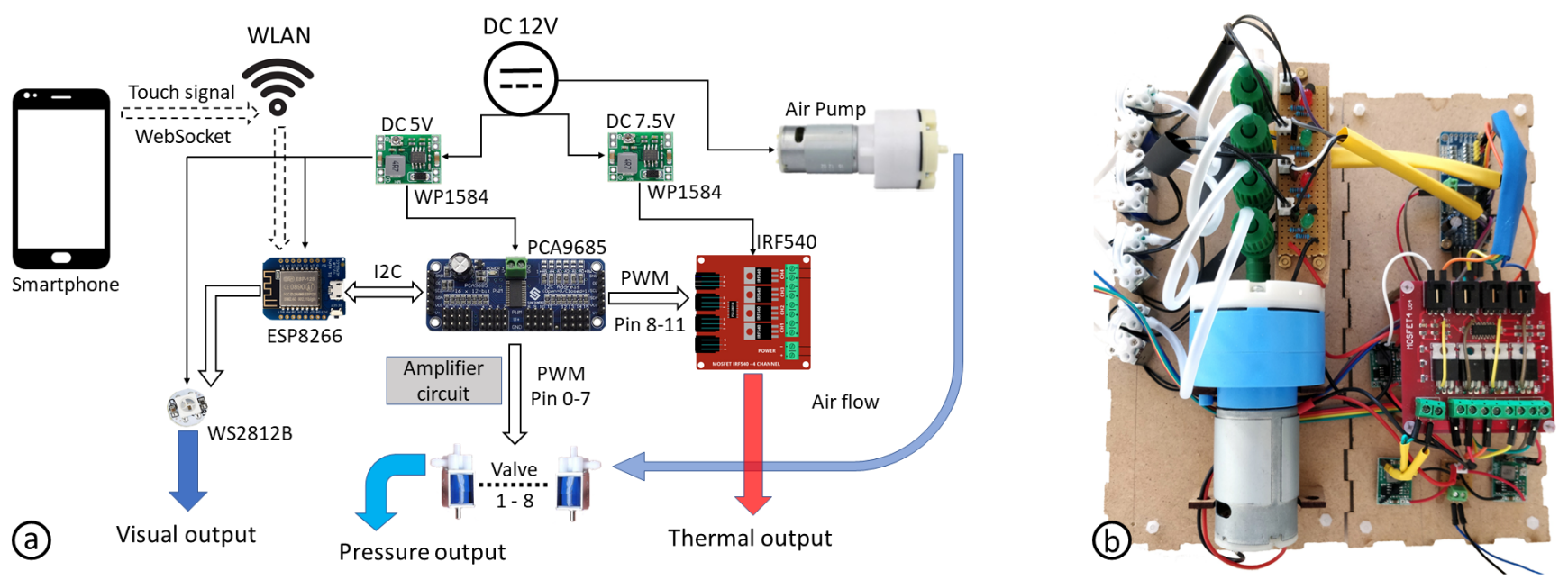

Figure 5: (a) Structure of the circuit and actuators. (b) Circuit and actuators.

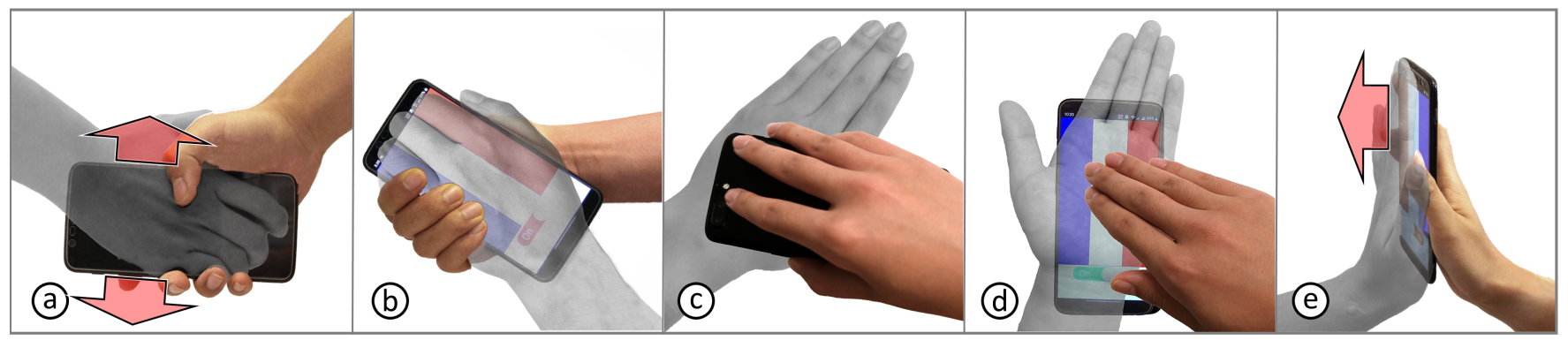

Figure 6: Hand-to-hand interactions with SansTouch. (a) Handshake. (b) Holding hands. (c) Tapping the back of the hand. (d) Touching the palm. (e) High-five.

driver (ref. PCA9685). A $12 \mathrm{~V}$ DC vacuum pump (ref. WP36C) as the air source to inflate airbags.

4.2.2 Body temperature. Past research highlighted the importance of conditioning a touch device to body temperature to generate a more natural and realistic touch sensation [17, 31]. To this end, we built a thermal layer with $0.1 \mathrm{~mm}$ Cr20Ni80 Nichrome wire and polyimide tapes. By adjusting the length of the Nichrome, we harmonized the resistances of these four thermal patches into $11 \pm 0.2 \Omega$. These four thermal patches are powered with $7.5 \mathrm{~V}$ DC through a MOSFET switch module (ref. IRF540) and controlled through the PWM servo driver. The temperature of the thermal patch can change from room temperatures to $45^{\circ} \mathrm{C}$, which is within the heat pain threshold [9].

4.2.3 Visual feedback. Previous works suggested that adding visual modality on remote touch devices could effectively enhance tactile perception [50,53]. Hence, we added one layer of addressable RGB LED (ref. WS2812b) to generate visual effects with different colors on top of the inflation layer. On the palm patch and the back patch, 4 LEDs are evenly distributed on the surface, while we included 2 LEDs on each of two side patches.

\subsection{Capturing input parameters with smartphones}

We developed a web interface that manages the communications between the smartphone and the wearable hand sleeve of SansTouch and controls all the parameters. We developed an Android application to detect the phone movement (with the accelerometer sensor and the device's orientation) and the touch events. By combining these input channels, we can generate a variety of hand-to-hand interactions (as described in Section 4.4). To increase the mobility of SansTouch, we used an ESP8266 micro controller (ref. Wemos D1 Mini) to control the actuators and to communicate with the android application or the web application via Wireless Local Area Network (WLAN) (Figure 5A). Thanks to the low latency of the WLAN, there is no significant delay between the input and the output, which largely ensures a fluid, synchronous touch interaction.

\subsection{Application: Mid-air hand-to-hand interactions with SansTouch}

We designed five simple hand-to-hand interaction samples to demonstrate the possibilities of using SansTouch to mediate touch 


\begin{tabular}{|c|c|c|c|c|c|c|}
\hline \multicolumn{3}{|c|}{ Input } & \multicolumn{4}{|c|}{ Output } \\
\hline Hand interaction & $\begin{array}{c}\text { Orientation of the } \\
\text { phone }\end{array}$ & Activate movement & Activated area(s) & $\begin{array}{c}\text { Activated } \\
\text { duration (ms) }\end{array}$ & $\begin{array}{c}\text { Intensity of } \\
\text { inflation and light }\end{array}$ & $\begin{array}{l}\text { Temperature } \\
\text { increase }\left({ }^{\circ} \mathrm{C}\right)\end{array}$ \\
\hline Handshake & $\begin{array}{l}y, z \text { axis: horizontally } \\
\text { positioned }\left( \pm 15^{\circ}\right)\end{array}$ & $\left|a_{x}\right| \geq 3,5 m s^{-2}$ & $\begin{array}{l}\text { Palm and two side: } \\
\text { (Figure 4a: (1)(2)(4)) }\end{array}$ & 2500 & $60 \%$ & 10 \\
\hline Holding hand & Any & $\begin{array}{c}3 \text { fingers touching on the } \\
\text { left/right side of the screen } \\
\text { (Figure } 6 c \text { ) }\end{array}$ & $\begin{array}{l}\text { Palm and two side: } \\
\text { (Figure 4a: (1)(2)(4)) }\end{array}$ & 4000 & $50 \%$ & 10 \\
\hline $\begin{array}{c}\text { Tapping the back } \\
\text { of the hand }\end{array}$ & $\begin{array}{l}z \text { axis: vertically } \\
\text { positioned }\left( \pm 15^{\circ}\right)\end{array}$ & $\left|a_{z}\right| \geq 3,5 m s^{-2}$ & $\begin{array}{l}\text { Back of the hand: } \\
\text { (Figure 4a: (3)) }\end{array}$ & 1000 & $50 \%$ & 10 \\
\hline Touching palm & Any & $\begin{array}{l}\text { Tapping the middle side of } \\
\text { the screen (Figure 5e) }\end{array}$ & Palm: (Figure 4a: (1)) & 1000 & $50 \%$ & 10 \\
\hline High-five & $\begin{array}{l}\text { y axis: vertically } \\
\text { positioned }\left( \pm 15^{\circ}\right)\end{array}$ & $\left|a_{z}\right| \geq 3,5 m s^{-2}$ & Palm: (Figure: 4a (1)) & 1000 & $100 \%$ & 10 \\
\hline
\end{tabular}

Figure 7: The parameters of the five pre-defined hand interactions. We set the parameters for the participants in the structured observational study to try them out. Users can easily adjust the parameters through the SansTouch's web interface.

communications, including handshakes and holding hands that are commonly used yet reduced in the survey's participants' current touch habits, as well as touching palms, tapping the back of the hand, and high-fives (Figure 6). After the connection between the smartphone and the controller is established, the touch stimuli can be triggered just by mimicking the hand movement as in real life, only now it is performed mid air while wearing the hand sleeve and holding the smartphone. We considered the Google developer guideline [18] to implement the interactions with SansTouch: the device coordinate frame, described by the values $x, y$, and $z$, is aligned based on the center of the device. The parameters are chosen as suggested in the literature $[17,31]$ as well as based our preliminary tests. The detailed parameters of these hand interactions as well as the output of each modality are listed in Figure 7.

\section{STUDY 2: STRUCTURED OBSERVATION ON SOCIAL TOUCH WITH SANSTOUCH}

Our next goal was to examine whether a mediated touch device like SansTouch could enhance social experience between users with semi-intimate relationships during a face-to-face communication while social distancing. This goal is complementary to the evaluations of touch devices in the literature that mainly focused on remote communications between users with intimate relationships. Our primary focuses were 1) to assess qualitatively how the participants experienced the unique condition of exchanging mediated touch for greetings during a face-to-face communication in an ecologically-valid setting; 2) to evaluate user perceptions of SansTouch's touch stimuli; and 3) to elicit user feedback regarding the generalizability aspects of SansTouch, including the use of SansTouch for other types of relationships and for remote communications. In order to do so, we conducted a structured observation in which the participants experienced exchanging greetings with and without SansTouch.

\subsection{Participants \& Setups}

Twelve participants took part in our study ( 3 women, 9 men, aged 20-37 years old, median age 28.3 years old), all worked/studied in the university. We followed the health protocol advised by WHO [33]. The participants sat in a wide meeting room with opened windows to ensure air circulation. We maintained a two-meter distance with an exception when the interviewer was setting up the SansTouch prototype on the participant's hand. We disinfected the surface of SansTouch device, the table, and the smartphone before starting each study session. Everyone involved in the study cleaned their hands and wore a mask upon entering the room.

\subsection{Method}

A study session consisted of three parts: an introductory interview; a scenario-based interaction; and an open-ended self exploration. We audio recorded the whole session, and video-recorded the hand interactions during the scenario-based interaction and the self exploration parts.

5.2.1 Introductory interview. We asked the participants to describe 1) their social touch habits before and after the lockdown; 2) the forms of social touch they reduced; and 3) social touch breakdown stories. The goal was to remind them of their experience related to social touch breakdowns with social distancing. We finished this part by setting up the SansTouch prototype on the participant's right hand and introducing how to exchange a mediated handshake with SansTouch. We let them familiarize with it for a few minutes, prompting them to think aloud. This part took around 15 minutes to complete.

5.2.2 Scenario-based interaction. Our goal was to compare the participant's experience when having a conversation in which the touch was 1) mediated (i.e., handshakes with SansTouch); and 2) substituted with other forms (i.e., waving hands, without SansTouch). Note that the context of the conversations is key in building a more concrete perception and experience. Therefore, we carefully 
chose a use case scenario that involved exchanging greetings with colleagues face to face, because 1) the interaction among colleagues in a meeting room was ecologically valid; and 2) our survey revealed more social touch breakdowns for semi-intimate relationships like colleagues, with handshakes being the most reduced. We substituted handshakes with waving hands since it was frequently mentioned in our survey results. We excluded other substitutions that still involved physical touch (e.g., bumping elbows) since we wanted to maintain the two-meter distance between people.

The use case scenario we introduced to the participants was: "You are attending the first group meeting after the summer holiday. Two of your colleagues, [colleague-1] and [colleague-2], are also attending the meeting. You have not met for a few months due to the summer holiday, so they would be happy to see you in person. They will enter the room and say hi. One of them also has the device as you do." To increase the ecological validity, we ran the study during the first two weeks after the university's official summer holiday. Three experimenters were involved: an interviewer leading the whole study session, and two (one man, one woman) posing as colleague-1 and colleague-2.

We used two SansTouch devices, one for the participant, one for the experimenter ${ }^{2}$. One colleague/experimenter opted for the mediated handshake with SansTouch to greet the participants, the other opted for waving hands. A OnePlus 5T smartphone was provided to the participants as the input device. The order of the two conditions was counter balanced along with the male and female experimenters, in order to avoid gender bias. We did not specifically brief the participants which condition would start first. The experimenters and the participants mainly had casual conversations such as "How are you?", "How was your holiday?", or "How do you feel getting back to work/school after some time?". The conversations lasted for 5 minutes in total.

After the conversations, the two experimenters left the room, and the participants were asked to compare their experience, specifically related to which condition made them feel 1) the social interaction was better; 2) it was easier to establish social connections with the colleagues. We also asked their perceptions of each modality of SansTouch. The interview took around 15 minutes.

5.2.3 Open-ended self exploration. Finally, we introduced all the five interaction samples we designed with SansTouch. We let the participants play around by themselves, prompting them to think aloud. The goal was to encourage them sharing how they would use it in their daily touch communication. We also asked about their perception of each interaction in terms of how realistic it was to the real touch, to probe on the generalizability of the SansTouch's interactions for other types of relationships and communications. For each interaction, we asked them to fill in a short Likert-scale question on how similar it was to the real touch (1="Completely different" to 5="Identical"). This part took around 15 minutes.

\subsection{Data analysis}

After transcribing the audio recording, we used thematic analysis to identify recurring themes [7]. We started with a round of open coding, where two authors tagged interesting and meaningful elements,

${ }^{2}$ The SansTouch's sleeve worn by the experimenters was not inflated - they wore it only to make the participants believed that the experimenters felt the touch sensation. built the codebook, then grouped the codes into categories. Finally, all authors iterated on the key themes during multiple meetings.

\section{KEY RESULTS FROM THE STRUCTURED OBSERVATIONAL STUDY}

\subsection{SansTouch enhanced interpersonal connections in face-to-face communication}

The participants reported social touch breakdowns that were largely consistent with those in the survey, including changes in their social touch habits and frustrations on having to re-establish their social touch habits. As such, almost all participants (11/12) preferred the mediated condition (i.e., greeting with SansTouch) during the face-to-face conversation, as they felt more positive interpersonal connections. Only one participant (P1) claimed no preference between the two conditions. Half of the participants (6/12) specifically mentioned that they felt a stronger connection with the person they were talking to after the handshake using SansTouch.

6.1.1 SansTouch encouraged behaviour transfer from physical to the mediated touch. Like during a real handshake, the majority of the participants (9/12) reported that they maintained eye contacts during the handshake with SansTouch, which led them feel that the mediated handshake was real and more engaging than just waving. P9 explained why: "The waving is friendly. But to establish the connection, the [touch] contact is also important, it makes people look at you, and you look at the people." Although some participants (3/12) felt that the physical distance made handshaking with SansTouch felt different from a real handshake, three participants still reported that using SansTouch made them feel as if the physical and the social distance were reduced. P12 explained that the mediated handshake made him unconsciously move his body closer to the experimenter: "With the device I feel less distance, maybe because I came closer [towards the person] to handshake". P4 also felt that SansTouch "established the proximity between people". Furthermore, P8 and P11 specifically stood up while shaking hands, but they stayed seated while waving. These results suggested that even with a physical distance, some behaviours from the physical touch (i.e., the real handshake) and its benefits for interpersonal communications were transferred into the mediated touch, including enhancing social bonding between people [16].

6.1.2 Synchronous interaction helped building the common ground in communication. The interaction design of SansTouch requires the two parties mimicking the hand position while moving their hands synchronously to trigger the handshake stimuli. The participants reported that they quickly understood the common ground of the communication just by seeing how the experimenters moved they hand as they reached out for a mediated handshake. This suggested that the participants could use SansTouch with minimum learning efforts.

Additionally, the behavioural alignment made the participants felt they were closer with the other person, as explained by P10, "With the device I feel closer to the person, because everything is synchronized, so I feel [we were] more on the similar thoughts." P11 also highlighted that the interaction with SansTouch affected his perception of how the other person would feel: "[The colleague] was also wearing the device, so I think she would feel what I felt. 
That made me feel a better connection with her." This highlights the importance of synchronicity between the movements of both users and the triggered touch stimuli.

\subsection{User perceptions of SansTouch}

6.2.1 Holding hands and handshakes most favorite, high-fives least. In the scenario-based interaction part, almost all participants (11/12) reported a strong preference on using SansTouch to greet as opposed to waving hands. Different descriptions were used to express their positive feelings using SansTouch: amazing (P7), fun (P9), natural (P1), surprising (P3, P8), unexpected and intense (P8). In particular, $\mathrm{P} 5$ reported "I'm actually feeling that she is shaking hands with me."

From the open-ended self exploration part, we found that among the five touch interactions, 10 out of 12 participants liked the mediated holding hands with SansTouch the most, while the other two participants preferred the mediated handshake. The holding-hand sensation was described as comfortable, pleasant (P1, P3) and enjoyable (P2). P3 said, "I really have the feeling that I am holding someone's hand. The sensation really lasts. I really establish a contact with the device [as if it were another person's hand]." The mediated high-five (9/12 participants) and patting the back of the hand (3/12) with SansTouch were their least favorite, mainly because 1) the delay and speed of the mediated high five (7/9 participants), as P8 mentioned "there is no pain, there is no sound"; 2) they rarely performed the interactions in real life (2/12). This is in line with the results from the Likert-scale questions related to how realistic each interaction was: mean/median 4.3/4.5 and 4.3/4 for holding hands and handshakes; 3.9/3 for high-five and touching palm; and 3.7/3 for patting the back of the hand. This highlights the importance of a touch device to generate not only a realistic touch sensation, but also other properties like its sound.

6.2.2 Warm pressure was key, visual feedback not so much. The majority of participants (10/12) perceived inflation as the most defining aspect of the interaction. P7 specifically said that the pressure was equally distributed, while P8 highlighted the inflation induced an intense sensation that was essential for mimicking handshakes. Almost half of the participants (5/12) specifically appreciated the warm sensation from the thermal layer. P9 highlighted that the combination of the inflation and the heat "recreated the feeling of real handshakes". However, P5 and P6 mentioned that they did not perceive the warmth during the conversation, as they mostly focused on maintaining eye contact. Since the participants were focusing on the conversation and maintaining eye contacts, none of them paid attention to the visual feedback (i.e., LED) while exchanging mediated handshakes. Only P2 and P5 noticed something was blinking, but then it was quickly ignored. P2 actually felt adding visual feedback made the mediated touch sensation even less similar to the real touch.

6.2.3 Holding a smartphone enhanced embodiment and usability. We observed that holding a smartphone was helpful in enhancing the embodiment of the hand interaction. Some participants $(4 / 12)$ described that for the mediated holding hands and handshakes, they felt like the smartphone was the hand of the other person. P10 compared the smartphone to a hand while handshaking in real life: he thought that the smartphone provided a constrain on how he should position his fingers and hand during the mediated handshake, in a similar way the other person's hand constrained his hand when he was gripping it in a real handshake. Additionally, $\mathrm{P} 4$ confirmed the accessibility of smartphone as a touch communication medium: "People already have their phones in their hand all day, so shaking the phone [to do the mediated handshake] can be learnt very easily." Related to usability, two participants were worried about throwing the phone while doing the mediated high-five. This suggested that the interaction design should also consider the practicality of the interaction (e.g., the device's safety).

\subsection{SansTouch in other context}

6.3.1 Beyond conventional social touch. The participants expressed interests in personalizing the mediated touch interactions. The examples given by the participants varied from wanting to customize the touch input parameters to creating new interactions such as "secret handshakes with lights" (P3). This suggested that SansTouch may not only serve as a replacement of conventional social touch, but also support the invention of new touch practices. Further, P10 mentioned:"This device is like a proxy, you don't really touch the person. Because there is no rule for these new devices, I can do the forms of touch that social norms usually do not allow, like touch or squeezing the hand of your boss." This suggested that by touching through SansTouch as a communication medium, the original meaning of the touch interaction might also evolve, which consequently might increase the expressivity of a form of social touch.

6.3.2 SansTouch for remote communications and different social relationships. In the context of face-to-face communications, half of the participants mentioned that they wanted to use SansTouch with friends and colleagues. This was mainly because they did not experience significant social touch breakdowns for intimate relationships while social distancing - a result that echoed our survey's result. That said, some participants (5/12) wanted to use SansTouch in remote communications with their partner and families. For example, P1 mentioned:"It would be good to hold [my partner's] hand and being held at the same time when I'm in my office." This suggested that SansTouch could be used for remote communications with other types of relationships. Finally, they also envisioned other contexts of use with SansTouch, including touching isolated people in hospitals (P3), Virtual Reality games (P9), tactile notifications (P11), and high-fives with athletes in a sport game (P11).

\section{DISCUSSION AND DESIGN IMPLICATIONS}

Our participants from both studies reported frustrations overcoming social touch breakdowns due to social distancing during the COVID-19 pandemic. Adopting new forms of touch (e.g., bumping elbow) was challenging, as it often involved awkward situation, requiring the participants to explicitly negotiate on the communication ritual. As such, the participants in our structured observational study saw SansTouch as a preferable alternative to replace the conventional social touch in face-to-face communications. They also perceived the touch sensation of SansTouch realistic (i.e., similar to the real touch) and pleasant. This suggested that SansTouch can generate realistic touch sensations without inducing uncanny valley issues [31, 44] or machine-like touch sensations [15]. 
While past research mostly focused on remote touch communications, our work highlights the needs to expand the design space of touch devices, by considering the device to be used in both contexts, especially because a touch interaction designed for remote communication might not be effective for face-to-face interaction, due to different reasons, as follow.

\subsection{Trade-off of combining different modalities}

When designing SansTouch, we followed design guidelines on touch devices from past studies focusing on remote touch communications, including combining different modalities such as touch and visual feedback $[43,50,53]$. Nevertheless, when evaluated in faceto-face communications, we found that the visual feedback was largely ignored since their attention was highly directed on the eyes and the facial expression of the other person. Thus, in the context of face-to-face communications, adding visual feedback to touch stimuli may interfere with the verbal communication, as it often relies on the visual modality as well. On the other hand, the comments related to missing high-five's sound point to a possibility of including audio feedback to touch communication tools. Designers should consider the balance between modalities and practicality in different mobility contexts.

\subsection{Synchronicity in face-to-face communication is key}

As highlighted in the results, synchronicity between the initiator's hand movement, the receiver's hand movement, and the touch stimuli generated by the touch device is critical for building a positive shared, bi-directional experience. As such, the interactions should be intuitive and easy to remember, to avoid delays and reduce the efforts for grounding the touch communication. This is especially critical if the touch communication tool will be used face-to-face in public spaces - users should be able to spontaneously start up the communication. On the other hand, delays and asynchronous interactions are more suitable for remote communications, perhaps even more desirable, for example if the users live in different time zones. To accommodate both contexts, designers should allow users to customize and personalize the touch interactions. Allowing customization would also help balancing between different modalities used in the touch interactions under different mobility contexts.

\subsection{Accessibility and social acceptability of the touch communication medium}

For face-to-face communications, designers should take into account the social acceptability of the touch medium when it is being used within relationships with different levels of intimacy or in public when strangers can see the interaction. The participants appreciated our design choice of combining a wearable device and a smartphone: the wearable was always there and the smartphone embodied the other person's hand. They also highlighted the accessibility and the social acceptance of grabbing smartphones in public. Another alternative solution is to develop extensions that can be easily embedded (e.g., [35]) or plugged into an existing smartphone (e.g., $[44,45])$. Considering how widespread smartphones are and the potential demand for mobile mediated touch communication, this could be a valuable direction towards developing a new type of mobile devices.

\section{LIMITATIONS AND FUTURE WORK}

Although our survey provides insights on social touch breakdowns, it is limited by our participant pool that did not include all cultural differences that may affect their perceptions towards touch communication habits.

Our device could also be improved in a number of ways to increase the real-life usability. We found that some touch interactions (especially high-fives) were found less convincing and could be improved by using more efficient hardware and tuning its parameters more finely. Some participants also mentioned that audio feedback could help in making the perception more realistic, especially for touch interactions that involve a quick strong contact like highfive. This feature, which has already been used in other studies for improving tactile illusions [22], is another promising direction for generating more realistic touch. We also consider replacing the solenoid valve and air pumps with liquid-to-gas actuators [46] to increase the portability and the real-life usability.

Finally, while the participants appreciated the technical design of SansTouch (e.g., smartphones embodying a hand), further investigation involving longitudinal, in-the-wild use of SansTouch is needed to reveal the real-life challenges of using a wearable sleeve with a smartphone to exchange touch. Future work should focus on building a more robust touch device, considering different mobility contexts, and evaluate it between two or more users in the wild.

\section{CONCLUSION}

This paper contributes a deeper understanding on social touch breakdowns with physical distancing due to COVID-19 pandemic and the challenges in establishing new touch habits. SansTouch enables users to touch others without direct physical contacts during a face-to-face communication with physical distancing. Our observational study showed that SansTouch enhanced interpersonal touch communication and social interactions, as well as encouraged behavioural transfer from a real interaction to mediated touch.

Our work points to future design directions for touch communication tools, highlighting the different design factors that designers and researchers should take into account when adapting remote touch communication tools under the context of face-to-face touch communication. We see combining touch devices with smartphones as a concrete step in enabling mediated touch communications that users can access anytime, anywhere, considering different social relationships.

\section{ACKNOWLEDGMENTS}

This work was supported by the French Agence Nationale de la Recherche (ANR-17-CE33-0006 SocialTouch). We thank Mickaël Bouhier, Michael Baker, and the DIVA group for their help and recommendations.

\section{REFERENCES}

[1] Leonardo Angelini, Maurizio Caon, Denis Lalanne, Omar Abou Khaled, and Elena Mugellini. 2014. Hugginess: Encouraging Interpersonal Touch through Smart Clothes. In Proceedings of the 2014 ACM International Symposium on 
Wearable Computers: Adjunct Program (Seattle, Washington) (ISWC '14 Adjunct). Association for Computing Machinery, New York, NY, USA, 155-162. https://doi.org/10.1145/2641248.2641356

[2] Sima Asadi, Nicole Bouvier, Anthony S Wexler, and William D Ristenpart. 2020. The coronavirus pandemic and aerosols: Does COVID-19 transmit via expiratory particles?

[3] Alexis E. Block and Katherine J. Kuchenbecker. 2018. Softness, Warmth, and Responsiveness Improve Robot Hugs. International fournal of Social Robotics 11, 1 (Oct. 2018), 49-64. https://rdcu.be/baa1k

[4] BusinessInsider.fr. 2020. Our ongoing list of how countries are reopening, and which ones remain under lockdown. Retrieved September 2, 2020 from https: //www.businessinsider.fr/us/countries-on-lockdown-coronavirus-italy-2020-3

[5] Mert Canat, Mustafa Ozan Tezcan, Celalettin Yurdakul, Eran Tiza, Buğra Can Sefercik, Idil Bostan, Oğuz Turan Buruk, Tilbe Göksun, and Oğuzhan Özcan. 2016. Sensation: Measuring the Effects of a Human-to-Human Social Touch Based Controller on the Player Experience. In Proceedings of the 2016 CHI Conference on Human Factors in Computing Systems (San Jose, California, USA) (CHI '16) Association for Computing Machinery, New York, NY, USA, 3944-3955. https: //doi.org/10.1145/2858036.2858418

[6] H. H. Clark and Susan E. Brennan. 1991. Grounding in Communication. In Perspectives on Socially Shared Cognition, Lauren Resnick, Levine B., M. John, Stephanie Teasley, and D. (Eds.). American Psychological Association, Washington, DC, 127-149. https://doi.org/10.1037/10096-006

[7] Victoria Clarke and Virginia Braun. 2014. Thematic Analysis. Springer New York, New York, NY, 1947-1952. https://doi.org/10.1007/978-1-4614-5583-7 311

[8] CNBC.com. 2020. World Health Organization warns: Coronavirus remains 'extremely dangerous' and 'will be with us for a long time'. Retrieved August 17 2020 from https://www.cnbc.com/2020/04/22/world-health-organzation-warnscoronavirus-will-be-with-us-for-a-long-time.html

[9] Ian Darian-Smith and Kenneth O Johnson. 1977. Thermal sensibility and thermoreceptors. Journal of Investigative Dermatology 69, 1 (1977), 146-153.

[10] Alexandra Delazio, Ken Nakagaki, Roberta L. Klatzky, Scott E. Hudson, Jill Fain Lehman, and Alanson P. Sample. 2018. Force Jacket: Pneumatically-Actuated Jacket for Embodied Haptic Experiences. In Proceedings of the 2018 CHI Conference on Human Factors in Computing Systems (Montreal QC, Canada) (CHI '18). Association for Computing Machinery, New York, NY, USA, 1-12. https: //doi.org/10.1145/3173574.3173894

[11] C. DiSalvo, F. Gemperle, J. Forlizzi, and E. Montgomery. 2003. The Hug: an exploration of robotic form for intimate communication. In The 12th IEEE In ternational Workshop on Robot and Human Interactive Communication, 2003. Proceedings. ROMAN 2003. IEEE, IEEE Press., New York, 403-408. https: //doi.org/10.1109/ROMAN.2003.1251879

[12] Beate Ditzen, Inga D Neumann, Guy Bodenmann, Bernadette von Dawans, Rebecca A Turner, Ulrike Ehlert, and Markus Heinrichs. 2007. Effects of different kinds of couple interaction on cortisol and heart rate responses to stress in women. Psychoneuroendocrinology 32, 5 (2007), 565-574. https: //doi.org/10.1016/j.psyneuen.2007.03.011

[13] Tiffany Field. 2010. Touch for socioemotional and physical well-being: A review. Developmental Review 30, 4 (2010), 367 - 383. https://doi.org/10.1016/j.dr.2011. 01.001

14] Kory Floyd. 2014. Relational and health correlates of affection deprivation Western fournal of Communication 78, 4 (2014), 383-403. https://doi.org/10.1080/ 10570314.2014.927071

[15] Masahiro Furukawa, Hiroyuki Kajimoto, and Susumu Tachi. 2012. KUSUGURI: A Shared Tactile Interface for Bidirectional Tickling. In Proceedings of the 3rd Augmented Human International Conference (Megève, France) (AH '12). Association for Computing Machinery, New York, NY, USA, Article 9, 8 pages. https://doi.org/10.1145/2160125.2160134

[16] Alberto Gallace and Charles Spence. 2010. The science of interpersonal touch an overview. Neuroscience and biobehavioral reviews 34,2 (2010), 246-259. https: //doi.org/10.1016/j.neubiorev.2008.10.004

[17] Daniel Gooch and Leon Watts. 2012. YourGloves, Hothands and Hotmits: Devices to Hold Hands at a Distance. In Proceedings of the 25th Annual ACM Symposium on User Interface Software and Technology (Cambridge, Massachusetts, USA) (UIST '12). Association for Computing Machinery, New York, NY, USA, 157-166. https://doi.org/10.1145/2380116.2380138

[18] google.com. 2020. Device Orientation and Motion. Retrieved September 10, 2020 from https://developers.google.com/web/fundamentals/native-hardware/deviceorientation\#when to use device orientation events

[19] Karen M Grewen, Bobbi J Anderson, Susan S Girdler, and Kathleen C Light. 2003. Warm partner contact is related to lower cardiovascular reactivity. Behavioral medicine 29, 3 (2003), 123-130. https://doi.org/10.1080/08964280309596065

[20] Antal Haans and Wijnand IJsselsteijn. 2006. Mediated social touch: a review of current research and future directions. Virtual Reality 9, 2-3 (2006), 149-159. https://doi.org/10.1007/s10055-005-0014-2

[21] Sabrina Hauser, Melinda J. Suto, Liisa Holsti, Manon Ranger, and Karon E. MacLean. 2020. Designing and Evaluating Calmer, a Device for Simulating Maternal Skin-to-Skin Holding for Premature Infants. In Proceedings of the 2020
CHI Conference on Human Factors in Computing Systems (Honolulu, HI, USA) (CHI '20). Association for Computing Machinery, New York, NY, USA, 1-15. https://doi.org/10.1145/3313831.3376539

[22] V. Hayward 2008. A brief taxonomy of tactile illusions and demonstrations that can be done in a hardware store. Brain Research Bulletin 75, 6 (2008), 742-752.

[23] Maria Henricson, Anders Ersson, Sylvia Määttä, Kerstin Segesten, and Anna-Lena Berglund. 2008. The outcome of tactile touch on stress parameters in intensive care: a randomized controlled trial. Complementary therapies in clinical practice 14, 4 (2008), 244-254. https://doi.org/10.1016/j.ctcp.2008.03.003

[24] Gijs Huisman, Aduen Darriba Frederiks, Betsy Van Dijk, Dirk Hevlen, and Ben Kröse. 2013. The TaSST: Tactile sleeve for social touch. In 2013 World Haptics Conference (WHC). IEEE, IEEE Press., New York, 211-216. https://doi.org/10. 1109/WHC.2013.6548410

[25] Stanley E Jones and A Elaine Yarbrough. 1985. A naturalistic study of the meanings of touch. Communications Monographs 52, 1 (1985), 19-56. https: //doi.org/10.1080/03637758509376094

[26] JoyHaptics. 2020. Feel Better With iXu Smart Bear - Remote touch solution for isolated people to feel closeness and touch. Retrieved August 17, 2020 from https://www.joyhaptics.com/

[27] Julia Katila, Yumei Gan, and Marjorie Goodwin. 2020. Interaction rituals and 'social distancing': New haptic trajectories and touching from a distance in the time of COVID-19. Discourse Studies 22, 4 (06 2020), 418-440. https://doi.org/10. $1177 / 1461445620928213$

[28] Dimitrios Kontaris, Daniel Harrison, Evgenia-Eleni Patsoule, Susan Zhuang, and Annabel Slade. 2012. Feelybean: Communicating Touch over Distance. In $\mathrm{CHI}$ '12 Extended Abstracts on Human Factors in Computing Systems (Austin, Texas, USA) (CHI EA '12). Association for Computing Machinery, New York, NY, USA, 1273-1278. https://doi.org/10.1145/2212776.2212439

[29] Sarah L Master, Naomi I Eisenberger, Shelley E Taylor, Bruce D Naliboff, David Shirinyan, and Matthew D Lieberman. 2009. A picture's worth: Partner photographs reduce experimentally induced pain. Psychological Science 20, 11 (2009), 1316-1318. https://doi.org/10.1111/j.1467-9280.2009.02444.x

[30] Masahiro Mori, Karl F MacDorman, and Norri Kageki. 2012. The uncanny valley [from the field]. IEEE Robotics \& Automation Magazine 19, 2 (2012), 98-100. https://doi.org/10.1109/MRA.2012.2192811

[31] Hideyuki Nakanishi, Kazuaki Tanaka, and Yuya Wada. 2014. Remote Handshaking: Touch Enhances Video-Mediated Social Telepresence. In Proceedings of the SIGCHI Conference on Human Factors in Computing Systems (Toronto, Ontario, Canada) (CHI '14). Association for Computing Machinery, New York, NY, USA, 2143-2152. https://doi.org/10.1145/2556288.2557169

[32] Shannon O'Brien and Florian 'Floyd' Mueller. 2006. Holding Hands over a Distance: Technology Probes in an Intimate, Mobile Context. In Proceedings of the 18th Australia Conference on Computer-Human Interaction: Design: Activities, Artefacts and Environments (Sydney, Australia) (OZCHI '06). Association for Computing Machinery, New York, NY, USA, 293-296. https://doi.org/10.1145/ 1228175.1228226

[33] World Health Organization. 2020. Coronavirus disease (COVID-19) advice for the public. Retrieved September 1, 2020 from https://www.who.int/emergencies/ diseases/novel-coronavirus-2019/advice-for-public

[34] Claudio Pacchierotti, Stephen Sinclair, Massimiliano Solazzi, Antonio Frisoli, Vincent Hayward, and Domenico Prattichizzo. 2017. Wearable haptic systems for the fingertip and the hand: taxonomy, review, and perspectives. IEEE transactions on haptics 10, 4 (2017), 580-600. https://doi.org/10.1109/TOH.2017.2689006

[35] Young-Woo Park, Kyoung-Min Baek, and Tek-Jin Nam. 2013. The Roles of Touch during Phone Conversations: Long-Distance Couples' Use of POKE in Their Homes. In Proceedings of the SIGCHI Conference on Human Factors in Computing Systems (Paris, France) (CHI '13). Association for Computing Machinery, New York, NY, USA, 1679-1688. https://doi.org/10.1145/2470654.2466222

[36] Young-Woo Park, Sungjae Hwang, and Tek-Jin Nam. 2011. Poke: Emotional Touch Delivery through an Inflatable Surface over Interpersonal Mobile Communications. In Proceedings of the 24th Annual ACM Symposium Adjunct on User Interface Software and Technology (Santa Barbara, California, USA) (UIST '11 Adjunct). Association for Computing Machinery, New York, NY, USA, 61-62. https://doi.org/10.1145/2046396.2046423

[37] Young-Woo Park, Chang-Young Lim, and Tek-Jin Nam. 2010. CheekTouch: An Affective Interaction Technique While Speaking on the Mobile Phone. In CHI '10 Extended Abstracts on Human Factors in Computing Systems (Atlanta, Georgia, USA) (CHI EA '10). Association for Computing Machinery, New York, NY, USA, 3241-3246. https://doi.org/10.1145/1753846.1753965

[38] Rosalind W Picard and Jennifer Healey. 1997. Affective wearables. Personal Technologies 1, 4 (1997), 231-240. https://doi.org/10.1007/BF01682026

[39] M Zia Sadique, W John Edmunds, Richard D Smith, William Jan Meerding, Onno De Zwart, Johannes Brug, and Philippe Beutels. 2007. Precautionary behavior in response to perceived threat of pandemic influenza. Emerging infectious diseases 13, 9 (2007), 1307. https://doi.org/10.3201/eid1309.070372

[40] Hooman Aghaebrahimi Samani, Rahul Parsani, Lenis Tejada Rodriguez, Elham Saadatian, Kumudu Harshadeva Dissanayake, and Adrian David Cheok. 2012. Kissenger: Design of a Kiss Transmission Device. In Proceedings of the Designing 
Interactive Systems Conference (Newcastle Upon Tyne, United Kingdom) (DIS '12). Association for Computing Machinery, New York, NY, USA, 48-57. https: //doi.org/10.1145/2317956.2317965

[41] Patrick Saunders-Hastings, James AG Crispo, Lindsey Sikora, and Daniel Krewski. 2017. Effectiveness of personal protective measures in reducing pandemic influenza transmission: A systematic review and meta-analysis. Epidemics 20 (2017) 1-20. https://doi.org/10.1016/j.epidem.2017.04.003

[42] Samarth Singhal, Carman Neustaedter, Yee Loong Ooi, Alissa N. Antle, and Brendan Matkin. 2017. Flex-N-Feel: The Design and Evaluation of Emotive Gloves for Couples to Support Touch Over Distance. In Proceedings of the 2017 ACM Conference on Computer Supported Cooperative Work and Social Computing (Portland, Oregon, USA) (CSCW'17). Association for Computing Machinery, New York, NY, USA, 98-110. https://doi.org/10.1145/2998181.2998247

[43] Jordan Tewell, Jon Bird, and George R. Buchanan. 2017. The Heat is On: A Temperature Display for Conveying Affective Feedback. In Proceedings of the 2017 CHI Conference on Human Factors in Computing Systems (Denver, Colorado, USA) (CHI '17). Association for Computing Machinery, New York, NY, USA 1756-1767. https://doi.org/10.1145/3025453.3025844

[44] Marc Teyssier, Gilles Bailly, Catherine Pelachaud, and Eric Lecolinet. 2018. MobiLimb: Augmenting Mobile Devices with a Robotic Limb. In Proceedings of the 31st Annual ACM Symposium on User Interface Software and Technology (Berlin, Germany) (UIST '18). Association for Computing Machinery, New York, NY, USA 53-63. https://doi.org/10.1145/3242587.3242626

[45] Marc Teyssier, Gilles Bailly, Catherine Pelachaud, Eric Lecolinet, Andrew Conn and Anne Roudaut. 2019. Skin-On Interfaces: A Bio-Driven Approach for Artificial Skin Design to Cover Interactive Devices. In Proceedings of the 32nd Annual ACM Symposium on User Interface Software and Technology (New Orleans, LA, USA) (UIST '19). Association for Computing Machinery, New York, NY, USA, 307-322. https://doi.org/10.1145/3332165.3347943

[46] Ryusei Uramune, Hiroki Ishizuka, Takefumi Hiraki, Yoshihiro Kawahara, Sei Ikeda, and Osamu Oshiro. 2020. HaPouch: Soft and Wearable Haptic Display Devices Using Liquid-to-Gas Phase Change Actuator. In Adjunct Publication of the 33rd Annual ACM Symposium on User Interface Software and Technology (Virtual Event, USA) (UIST'20 Adjunct). Association for Computing Machinery, New York, NY, USA, 53-55. https://doi.org/10.1145/3379350.3416183
[47] Jan BF Van Erp and Alexander Toet. 2015. Social touch in human-computer interaction. Frontiers in digital humanities 2 (2015), 2. https://doi.org/10.1109/ WHC. 2007.92

[48] Rongrong Wang and Francis Quek. 2010. Touch \& Talk: Contextualizing Remote Touch for Affective Interaction. In Proceedings of the Fourth International Conference on Tangible, Embedded, and Embodied Interaction (Cambridge, Massachusetts, USA) (TEI '10). Association for Computing Machinery, New York, NY, USA, 13-20. https://doi.org/10.1145/1709886.1709891

[49] Sheryle J Whitcher and Jeffrey D Fisher. 1979. Multidimensional reaction to therapeutic touch in a hospital setting. Fournal of Personality and Social Psychology 37, 1 (1979), 87. https://doi.org/10.1037/0022-3514.37.1.87

[50] Graham Wilson and Stephen A. Brewster. 2017. Multi-Moji: Combining Thermal, Vibrotactile \& Visual Stimuli to Expand the Affective Range of Feedback. In Proceedings of the 2017 CHI Conference on Human Factors in Computing Systems (Denver, Colorado, USA) (CHI '17). Association for Computing Machinery, New York, NY, USA, 1743-1755. https://doi.org/10.1145/3025453.3025614

[51] Yohei Yamaguchi, Hidekatsu Yanagi, and Yoshinari Takegawa. 2013. Touch-shake: Design and implementation of a physical contact support device for face-to-face communication. In 2013 IEEE 2nd Global Conference on Consumer Electronics (GCCE). IEEE, IEEE Press., New York, 170-174. https://doi.org/10.1109/GCCE. 2013.6664789

[52] Eric M Young, Amirhossein H Memar, Priyanshu Agarwal, and Nick Colonnese. 2019. Bellowband: A Pneumatic Wristband for Delivering Local Pressure and Vibration. In 2019 IEEE World Haptics Conference (WHC). IEEE, IEEE Press., New York, 55-60. https://doi.org/10.1109/WHC.2019.8816075

[53] Zhuoming Zhang, Robin Héron, Eric Lecolinet, Françoise Detienne, and Stéphane Safin. 2019. VisualTouch: Enhancing Affective Touch Communication with MultiModality Stimulation. In 2019 International Conference on Multimodal Interaction (Suzhou, China) (ICMI '19). Association for Computing Machinery, New York, NY, USA, 114-123. https://doi.org/10.1145/3340555.3353733

[54] Mengjia Zhu, Amirhossein H. Memar, Aakar Gupta, Majed Samad, Priyanshu Agarwal, Yon Visell, Sean J. Keller, and Nicholas Colonnese. 2020. PneuSleeve: In-Fabric Multimodal Actuation and Sensing in a Soft, Compact, and Expressive Haptic Sleeve. In Proceedings of the 2020 CHI Conference on Human Factors in Computing Systems (Honolulu, HI, USA) (CHI '20). Association for Computing Machinery, New York, NY, USA, 1-12. https://doi.org/10.1145/3313831.3376333 